\title{
Behavior of beam on elastic foundation using element free Galerkin method
}

\author{
H.T.T. Lan* \\ Department of Civil Engineering, University of Architecture HCMC, 196 Pasteur Street, District 3, HCM City, VIETNAM \\ "Corresponding Author's e-mail: tltechonlinesom@gmail.com, Tel +84-028-38.222748 \\ ORCID iD: https://orcid.org/0000-0002-5195-1856
}

\begin{abstract}
One of mesh free methods, element free Galerkin method, is presented to analyze the finite beam on elastic foundation. The shape functions are constructed by using the moving least square interpolation based on a set of nodes that are arbitrarily distributed in specified domain. Discrete system equations are derived from the variation form of system equations. Numerical examples of finite beam on elastic foundation are given by establishing Matlab code. The results of this paper demonstrate the effectiveness of the proposed method with small errors compared to analytical solutions.
\end{abstract}

Keywords: mesh free method, element free Galerkin method, moving least square, finite beam, elastic foundation.

DOI: http://dx.doi.org/10.4314/ijest.v12i4.2

Cite this article as:

Lan H.T.T.. 2020. Behavior of beam on elastic foundation using element free Galerkin method. International Journal of Engineering, Science and Technology, Vol. 12, No. 4, pp. 14-22. doi: 10.4314/ijest.v12i4.2

Received: October 8, 2020; Accepted: October 26, 2020; Final acceptance in revised form: October 28, 2020

\section{Introduction}

The use of the finite element method (FEM) of analysis is very common nowadays. This technique has been widely used with great accuracy but as the complexity of the shape domain increases, this accuracy becomes an issue. Since this method requires the presence of a predefined mesh for proper analysis to be carried, modeling of structures with complicated geometries requires a very fine mesh arrangement. Some of the major disadvantages of FEM are as follows: FEM mesh construction is very costly as most of the time used for analysis is consumed by mesh construction; Accuracy of the stress obtained is less. The entire mesh must be refined from the start when a desired accuracy is not achieved, thus making all the previous works a waste. A lot of research has been done to develop FEM or an alternative to FEM such as (Hieu et al., 2017), (Ton et al., 2020), (Liu et al., 2007), and so on. Liu (2002) presented the mesh-free method as a method without the use of any predefined mesh for problem domain. Recently, this method is extended in the area of computational mechanics. There are different versions of the mesh free methods (MFMs) developed so far to analyse the stress and displacement in the solid. A mesh free method is a method that represent the problem domain by set of scattered nodes in its. These nodes do unlike nodes in the other numerical methods. Mesh free method is a new numerical analysis method having excellent accuracy and rapid convergence. Moving least square (MLS) approximation is given in Galerkin method which is developed by (Nyroles et al., 1992) for solving partial differential equations.

After them, the method has been modified and refined by (Belytschko et al., 1994) and named as element free Galerkin (EFG) method. In this method, the MLS interpolants were used to construct the trial and test functions for the variational principle (weak form) and weight functions. The point interpolation method (PIM) was given by (Liu et al., 1999) to construct shape functions which possess Kronecker delta property. Direct nodal integration, on the other hand, leads to a numerical instability due to under integration and vanishing derivatives of shape functions at the nodes. Another new approach is developed in (Chen $e t$ al., 2001) as well as a new mesh free method which is the PIM based on radial basis function (RPIM) is given in (Zhang et al., 2008). The 
analysis of thin plate of complicated shape using a mesh free method is presented in (Liu et al., 2001). Method uses MLS interpolation to construct shape functions based on a set of nodes arbitrarily distributed in the analysis domain. Penalty method was employed to enforce the essential boundary conditions for static analysis. Similar type of formulation is used in (Dai et al., 2004) for the analysis of static deflection and natural frequencies of thin and thick laminated composite plates using high order shear deformation theory. Enforcing essential boundary conditions in Galerkin based mesh free methods is given in (Fernandez et al., 2004).

A new method is given in (Xin et al., 2004) of deriving the particular solutions of differential equations by using the Sloan hyper-interpolation and fast Fourier transform. The special feature of their approach is that a close form of particular solutions can be easily obtained and the matrix formulation for evaluating particular solution is not required. The mesh free method was extended for the elastoplastic analysis of reinforced soils as given in (Binesh et al., 2010). The radial point interpolation method was used to construct the shape functions in the applied mesh free method which has the ability of modeling slippage between the soil and rein-forcement. Comparison of the computational complexity of the meshless local Petrov Galerkin method (MLPG) with the finite difference method (FDM) and finite element methods (FEM) is presented in (Trobec et al., 2009) from the user point of view. According to them, MLPG is the most complex of the three methods. Aspect of consistency, stability, explicit time integration and efficiency within the context of nodal integration is presented by (Puso et al., 2008). A generalized mesh free approximation suggested in (Wu et al., 2011) by introducing an enriched basis function in the original Shepard's method to meet the linear or higher order reproducing conditions. It is analogous to a special root finding scheme of constraint equations which enforces improvement in the basis functions and the reproducing conditions with certain orders within a set of nodes.

A few papers with deep mathematical knowledge are also cited as essential basis for programming (Turuna et al., 2020), (Duressa et al., 2019, 2022). From the reviewed literature it can be seen that there has been a great deal of research into the application of MFMs in different fields of science. Very few works have been reported on the application to the problems of beams on elastic foundation. In this study, the behavior of beam on elastic foundation is presented by using element free Galerkin (EFG) method which is a one type of mesh free method. The EFG method presented employs generalized moving least square approximation to generate the shape functions and the essential boundary conditions are enforced directly at each constraint boundary point. A parametric study is performed to investigate the effect of few selected parameters.

The next sections of this paper are as follows. The brief of element free Galerkin (EFG) method, including the MLS approximation and the Galerkin weak form, is presented in Sect. 2. Two basic types of elastic foundations and the governing differential equation of the beam on elastic foundation are shown in Sect. 3. Not only point load but also uniformly distributed load are considered in this section. And the numerical results of this paper are compared with analytical solution to prove the effectiveness of proposed method. Some concluding remarks are given in the last section.

\section{Brief of element free Galerkin (EFG) method}

The element free Galerkin (EFG) method is one of the mesh free methods which have been developed in (Belytschko et al., 1994). In EFG method, the MLS approximation and the Galerkin weak form are used to establish the shape functions as well as to develop the discretized system equations. The high order polynomials for shape functions are common used but even linear polynomial based functions give quite accurate results for the curved boundaries which are represented by nodes. For some mesh free methods such as the EFG method, a background mesh is needed to be used in integration of the system matrices. However, the shape of the background mesh is not strict, provided that accuracy in integrations is adequate. It should be noted that the moving least squares approximation is based only on the information of the values of the variables at some scattered points.

The EFG method is presented in this paper which employs the generalized moving least square approximants to approximate the function and the transformation matrix for imposing the essential boundary conditions. The moving least square (MLS) is widely used to generate the shape functions for various mesh free methods. Let us consider a displacement function $u(\mathrm{x})$ on the domain as shown in Fig. 1, the approximated value of $u(\mathrm{x})$ can be represented as

$$
u(x)=p_{j}(x) a_{j}(x) .
$$

where $a(x)$ and $p(x)$ represent the coefficient matrix and the polynomial matrix. The Pascal's triangle is used to choice the polynomial function. For example, a $1 \mathrm{D}$ bar having a linear basis equal 2 , the polynomial matrix $p(x)=[1, x]$. Whereas for a $2 \mathrm{D}$ element having linear basis equal 3, the matrix will be $p(x)=[1, x, y]$. For cases in which deflection must be found a quadratic polynomial is used as continuity up to the second derivative of the shape function needs to be established for consistency. Therefore, author takes basis equal 6 and $p(x)=\left[1, x, y, x^{2}, y^{2}, x y\right]$. The coefficients are determined using the values at the field nodes represent inside the support domain. The support domain may be defined as the small area neighboring a point inside which the field nodes exert influence over the point of consideration. Moreover, the approximation is generally weighted to maintain continuity in the movement of nodes in as well as out of the support domain and to increase the accuracy of the interpolation function. 


$$
J=\sum w\left(x-x_{I}\right)\left[u^{h}\left(x-x_{I}\right)-u_{I}(x)\right] .
$$

with $u_{I}$ is the value of the nodal displacement and $w\left(x-x_{i}\right)$ denotes the weight function. The coefficient matrix $a(x)$ must be chosen in such a way that the weighted residual is minimum

$$
\frac{\delta J}{\delta a}=0 .
$$

Hence the following linear equations are obtained

$$
A(x) a(x)=B(x) u_{s},
$$

In which

$$
A(x)=\sum w(x) p\left(x_{I}\right) p^{T}\left(x_{I}\right),
$$

and

$$
B(x)=w(x) p\left(x_{I}\right)
$$

Solving the above equations, author obtain

$$
a(x)=A^{-1}(x) B(x) u_{s} .
$$

and the shape function

$$
\Phi(x)=\sum p(x) A^{-1}(x) B(x)=p^{T} A^{-1} B .
$$

Let us consider $A(x) \gamma(x)=p(x)$ therefore $\Phi(x)=\gamma^{T}(x) B(x)$. Some of the weight functions are listed as cubic spline weight function and quartic spline weight function.

$$
\begin{gathered}
w_{1}(s)=\left\{\begin{array}{cc}
\frac{2}{3}-4 s^{2}+4 s^{3} & \text { if }|s| \leq \frac{1}{2} \\
\frac{4}{3}-4 s+4 s^{2}-\frac{4}{3} s^{3} & \text { if } \frac{1}{2}<|s| \leq 1 \\
0 & \text { if }|s|>1
\end{array}\right. \\
w_{1}(s)=\left\{\begin{array}{cc}
1-6 s^{2}+8 s^{3}-3 s^{4} & \text { if }|s| \leq 1 \\
0 & \text { if }|s|>1 .
\end{array}\right.
\end{gathered}
$$

with $s=\frac{\left\|x-x_{I}\right\|}{d m_{I}}$ is a radius of influence and $d m_{I}$ is a size of the domain of influence of the $I^{\text {th }}$ node. For example, we consider two case of the support domain. 


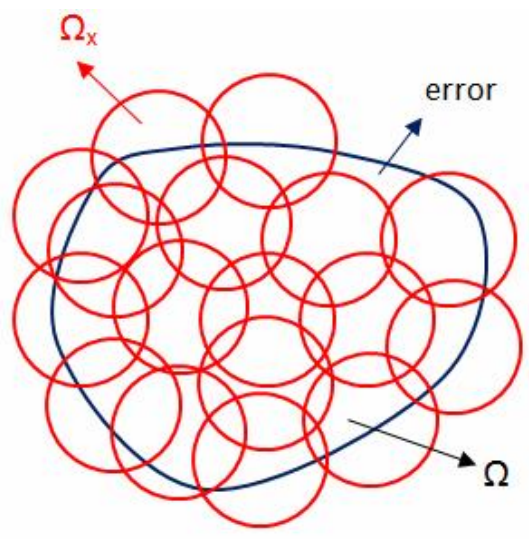

(a)

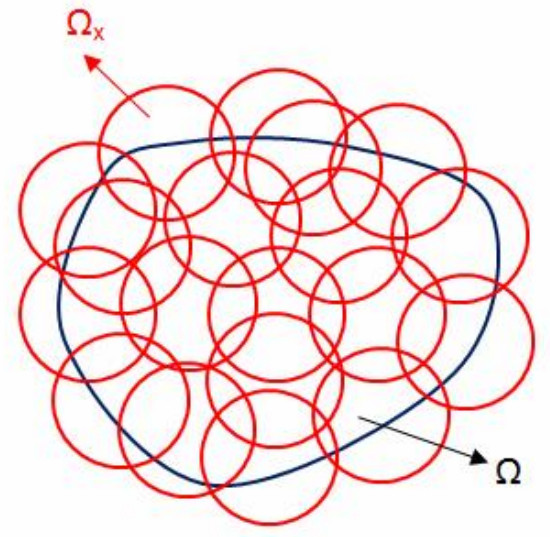

(b)

Figure 1. These cases are error or good because of the compact support

In Fig. 1a, the domain of problem did not cover entire domain, because the radius of support domain does not enough large to overlap the area of problem domain. The solution in this case is singular and we can see the support enough large to overlap entire domain of problem, the result is good in Fig. 1b. The Galerkin weak form in statics for a domain is given by

$$
\int_{\Omega} \delta(\mathbf{L u})^{T} \mathbf{c}(\mathbf{L u}) d \Omega-\int_{\Omega} \delta \mathbf{u}^{T} \mathbf{b} d \Omega-\int_{\Gamma_{t}} \delta \mathbf{u}^{T} \mathbf{t} d \Gamma=0 .
$$

The governing relation for the EFG method can be obtained by using the MLS approximation and the Galerkin weak form

$$
\mathbf{L u}=\sum \mathbf{B u} .
$$

The shape functions achieved by the MLS approximation do not satisfy the Kronecker delta property, therefore it is impossible for the shape function to satisfy the essential boundary conditions. Hence author uses Lagrange multipliers $\lambda$ to satisfy the boundary conditions. Now the nodal values of the Lagrange function are known, thus by means of Lagrange interpolation, its values are determined and the final discretized equations for are obtained. The final discretized equations of the EFG method are as follows

$$
\begin{gathered}
\mathbf{K} \mathbf{u}+\mathbf{G} \lambda-\mathbf{F}=0, \\
\mathbf{G}^{\mathrm{T}} \mathbf{u}-\mathbf{q}=0 .
\end{gathered}
$$

with

$$
\begin{gathered}
\mathbf{K}_{I J}=\int_{I K} \mathbf{B}_{I}^{T} \mathbf{C B}_{J} \mathrm{~d}, \\
\mathbf{G}_{I K}=-\int_{\Gamma_{U}} \Phi_{I} \mathbf{N}_{K} d \Gamma, \\
\mathbf{F}_{I}=\int_{\Gamma_{T}} \Phi_{I} \overline{\mathbf{t}} d \Gamma+\int_{\Omega} \Phi_{I} \mathbf{b} d \Omega, \\
\mathbf{q}_{K}=-\int_{\Gamma_{U}} \mathbf{N}_{K} \overline{\mathbf{u}} d \Gamma .
\end{gathered}
$$

where

$$
\begin{gathered}
\mathbf{B}_{I}=\left[\begin{array}{cc}
\Phi_{I, x} & 0 \\
0 & \Phi_{I, y} \\
\Phi_{I, y} & \Phi_{I, x}
\end{array}\right], \\
\mathbf{N}_{K}=\left[\begin{array}{cc}
N_{K} & 0 \\
0 & N_{K}
\end{array}\right] .
\end{gathered}
$$


The EFG method requires a background mesh for the integration purpose. Mesh just subdivides the domain into chosen shape and there is no influence of this mesh in the formation of the shape function as is the case with FEM. More information and formulas related to this method can be found in (Belytschko et al., 1996).

\section{Beam on elastic foundation}

Based on related theory, there are two basic types of elastic foundations. The first type is indicated by the fact that the pressure in the foundation is proportional at every point to the deflection occurring at that point and is independent of deflections produced elsewhere in the foundation. The second type is characterized by the elastic solid, which represents the case of complete continuity in the supporting medium in contrast with the first type. The concept of beam on elastic foundation can be extended in different engineering fields. The Winkler foundation with an infinite number of linear spring $k_{s}$ is used to describe the elastic support. The governing differential equation of the beam with constant flexural rigidity EI can be written as follows (Hetenyi, 1958):

$$
E I \frac{d^{4} u}{d x^{4}}+k_{s} u=q(x)
$$

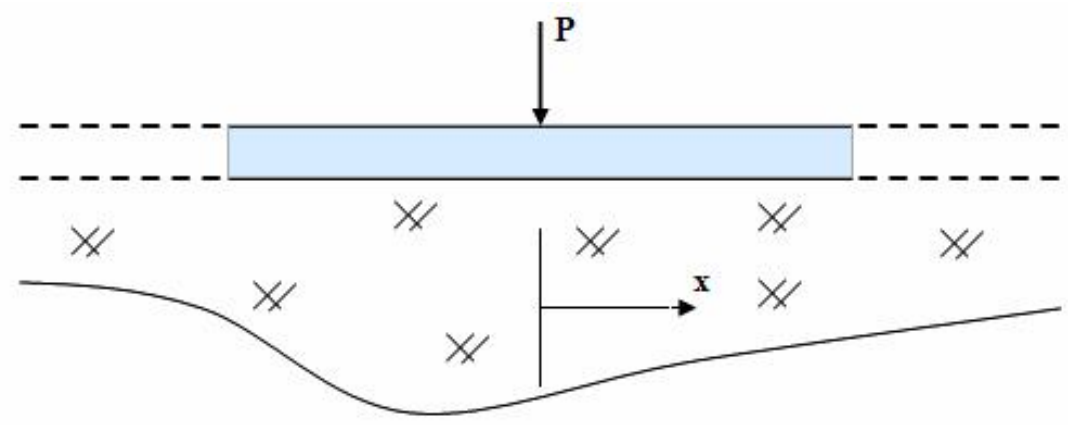

Figure 2. Beam on elastic foundation with central point load

If point load $P$ is acting at centre of beam as shown in Fig. 2 then two notions of boundary condition should be considered as follows: slope at the location of point load, $\theta=0$ and shear force at the location of point load, $V=-0.5 P$. The analytical solution of the above equation can be found in the many articles listed as follows (Dobromir, 2012), (Binesh et al., 2010, 2012). Besides, a computer program based on the formulation of EFG method with cubic spline weight function and circular supports is developed in Matlab by author as appendix section.

For a specific problem, a beam of having flexural rigidity of $E I=20000 \mathrm{kN} / \mathrm{m}^{2}$ subjected to central point load $P=100 \mathrm{kN}$ is considered. However, nodes are considered upto distance $L$ of $40 \mathrm{~m}$ and $60 \mathrm{~m}$ from centre. Soil modulus $k_{s}=2000 \mathrm{kN} / \mathrm{m}^{3}$ was considered and the value of $q(x)$ equals to zero for this example. Number of field nodes was varied as 6 for case 1 and 11 for case 2 in the present study to examine their effect on prediction. Results obtained from this study are represented in Table 1 and Table 2 as well as in Fig. 3 and Fig. 4 respectively.

Table 1. Displacements at nodes and analytical solutions with $L=40 \mathrm{~m}$ from centre

\begin{tabular}{cccccc}
\hline & \multicolumn{5}{c}{ Displacement } \\
\hline 6 nodes & -0.02389 & 0.00211 & -0.00069 \\
11 nodes & -0.02749 & -0.00409 & 0.00191 & 0.00070 & -0.00061 \\
Analytical & -0.02800 & -0.00415 & 0.00189 & 0.00070 & -0.00060 \\
\cline { 2 - 5 } & \multicolumn{5}{c}{$(\mathrm{mm})$} \\
\cline { 2 - 5 } & -0.00011 & -0.00012 & -0.00171 \\
& -0.00010 & -0.00007 & -0.00070 & -0.00190 \\
& & 4 & -0.00190 \\
\hline
\end{tabular}


Table 2. Displacements at nodes and analytical solutions with $L=60 \mathrm{~m}$ from centre

\begin{tabular}{|c|c|c|c|c|c|c|c|}
\hline \multicolumn{8}{|c|}{ Displacement } \\
\hline 6 nodes & -0.01849 & & 0.00188 & & -0.00091 & & -0.00029 \\
\hline 11 nodes & -0.01519 & -0.00271 & 0.00119 & 0.00070 & -0.00060 & 0.00041 & -0.00011 \\
\hline \multirow[t]{5}{*}{ Analytical } & -0.01500 & -0.00265 & 0.00110 & 0.00070 & -0.00060 & 0.00041 & -0.00010 \\
\hline & & \multicolumn{4}{|c|}{$(\mathrm{mm})$} & & \\
\hline & & & -0.00012 & & -0.00018 & & \\
\hline & & -0.00007 & -0.00008 & -0.00020 & -0.00010 & & \\
\hline & & -0.00006 & -0.00007 & -0.00020 & -0.00010 & & \\
\hline
\end{tabular}

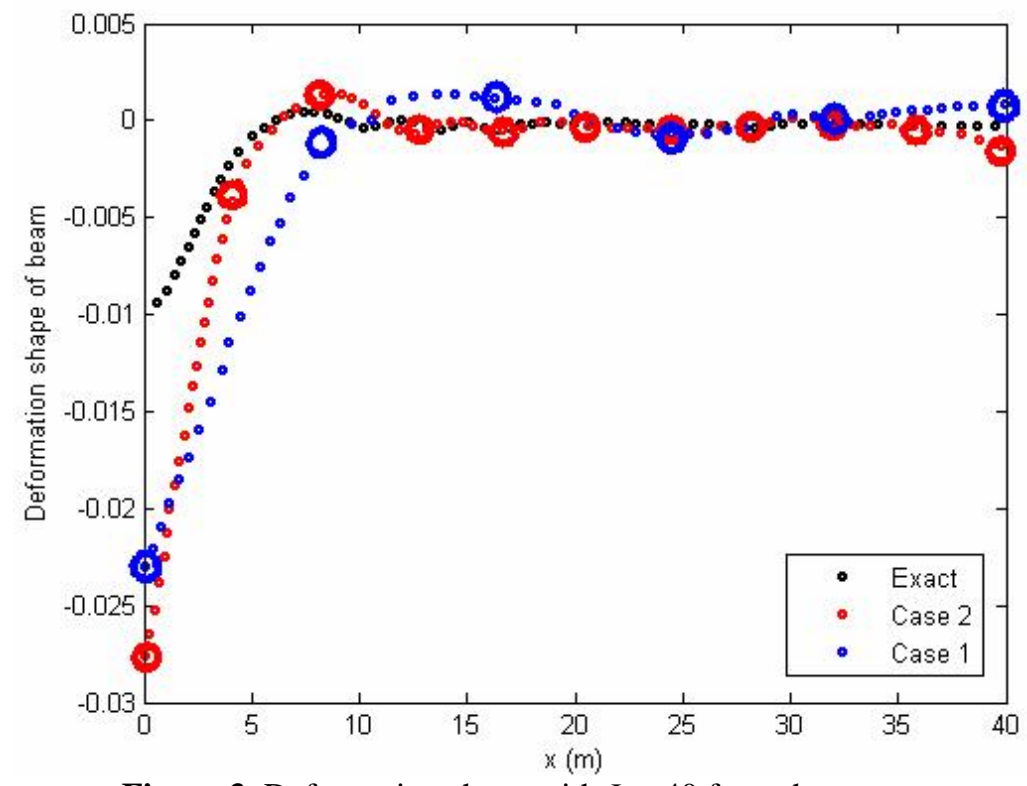

Figure 3. Deformation shape with $L=40$ from the center

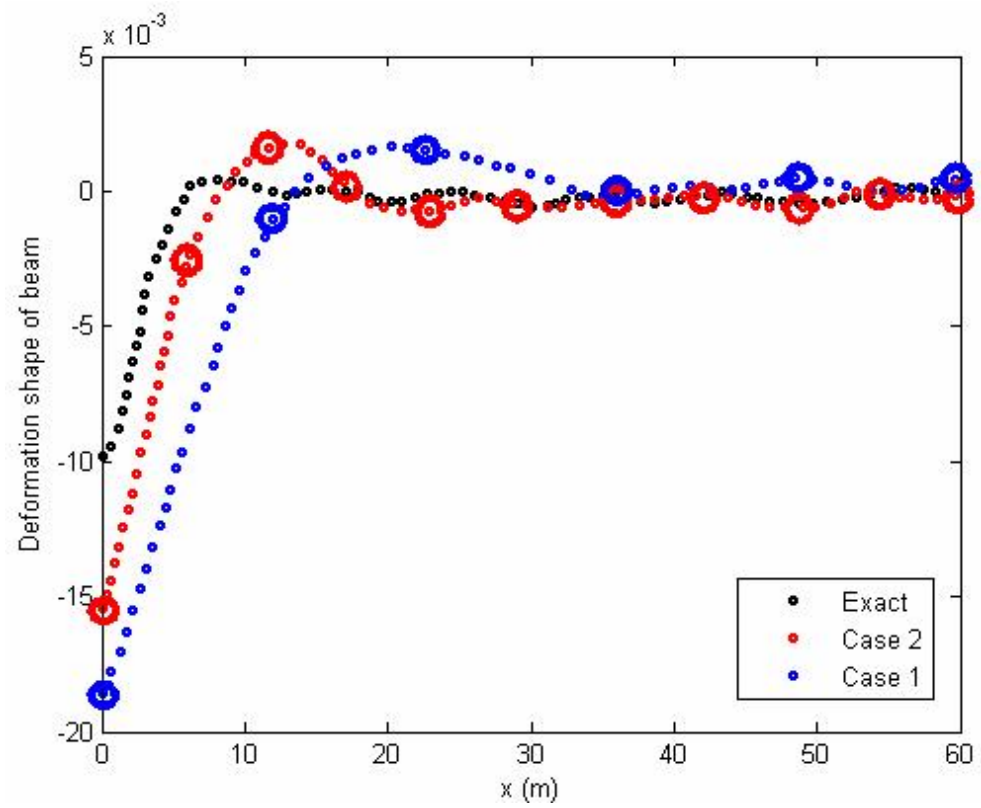

Figure 4. Deformation shape with $L=60$ from the center 
Finally, consider a beam with uniformly distributed load $q=1000 \mathrm{kN} / \mathrm{m}$ as shown in Fig. 5. Similar to above example, nodes are considered upto distance $L$ of $10 \mathrm{~m}$ from centre. Results obtained from this study are compared with analytical solution as Fig. 6 respectively.

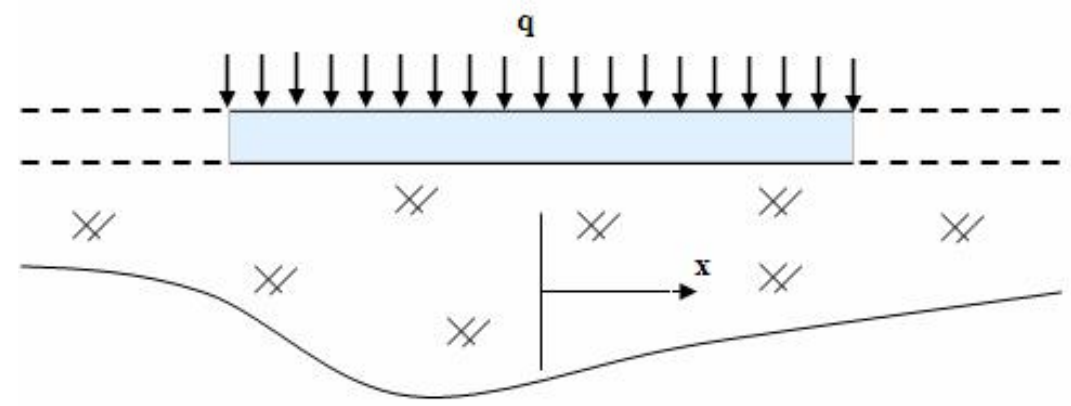

Figure 5. Beam on elastic foundation with uniformly distributed load

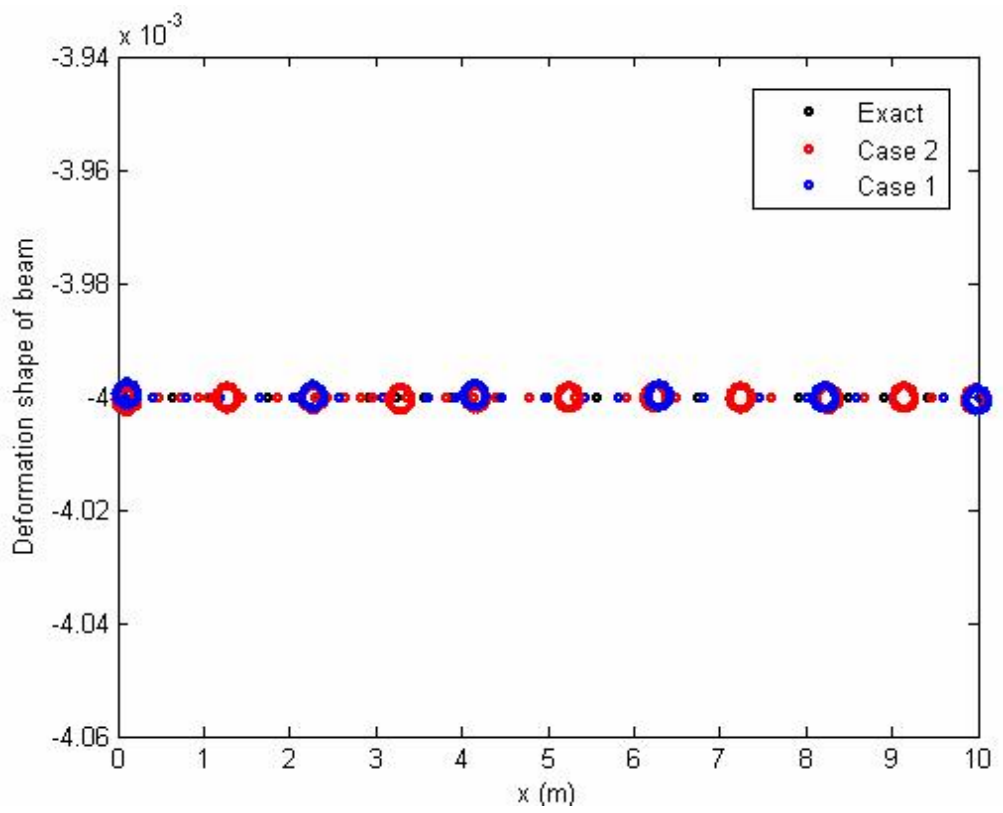

Figure 6. Deformation shape with $L=10$ from the center

\section{Conclusions}

In the present work, a Matlab code has been developed to analyse finite beam on elastic foundation with central point load by EFG method. The accuracy and convergence of the meshless method in predicting the displacement along the length of beams are examined. From the observed results in can be concluded that the mesh free method can be used efficiently for the analysis of the finite beam on elastic foundation. Displacements are taken as field variables. Unlike the finite element method, the meshless method requires no structured mesh, since only a scattered set of nodal points is required in the domain of interest. The proposed results are good agree with analytical solution. 


\section{Appendix}

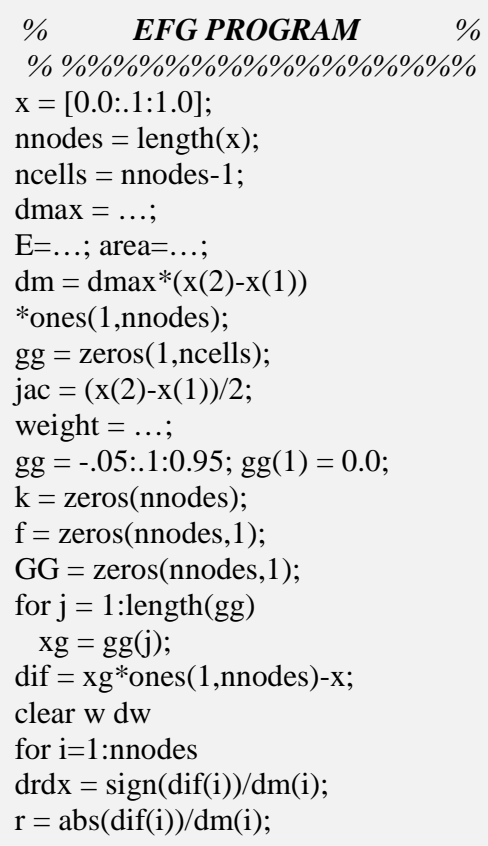

\section{Main MatLab Code}

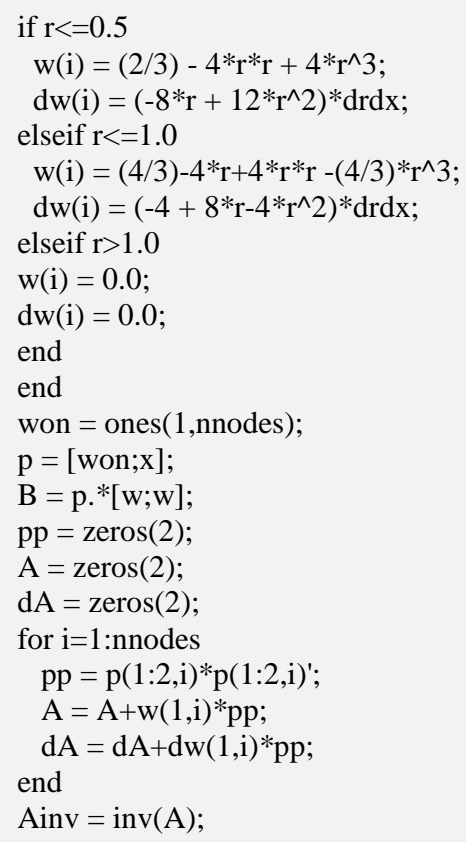

\section{Acknowledgement}

The author would like to thank the anonymous referee whose comments improved the original version of this manuscript.

\section{References}

Belytschko T., Lu Y.Y. and Gu L., 1994. Element free Galerkin methods. International Journal for Numerical Methods in Engineering, Vol.37, No.2, pp.229-256. https://doi.org/10.1002/nme.1620370205

Belytschko T., Krongauz Y., Organ D., Fleming M. and Krysl P., 1996. Meshless methods: An overview and recent developments. Computer Methods in Applied Mechanics and Engineering, Vol.139, No.1-4, pp.3-47. https://doi.org/10.1016/S00457825(96)01078-X

Binesh S.M., Hataf N. and Ghahramani A., 2010. Elasto plastic analysis of reinforced soils using mesh free method. Applied Mathematics and Computation, Vol.215, pp.4406-4421. doi:10.1016/j.amc.2010.01.004

Binesh S.M., 2012. Analysis of beam on elastic foundation using the radial point interpolation method. Scientia Iranica A, Vol. 19, No. 3, pp.403-409. https://doi.org/10.1016/j.scient.2012.04.003

Chen J.S., Wu C.T., Yoon S. and You Y., 2001. A stabilized conforming nodal integration for Galerkin mesh-free methods. International Journal for Numerical Methods in Engineering, Vol. 50, pp.435-466. doi.org/10.1002/10970207(20010120)50:2<435::AID-NME32>3.0.CO;2-A

Dai K.Y., Liu G.R., Lim K.M. and Chen X.L., 2004. A mesh-free method for static and free vibration analysis of shear deformable laminated composite plates. Journal of Sound and Vibration, vol. 269, pp. 633-652. https://doi.org/10.1016/S0022460X(03)00089-0

Dobromir D., 2012. Analytical solution of beam on elastic foundation by singularity functions. Engineering Mechanics, Vol.19, No.6, pp.381-392.

Fernandez S.M., Huerta A., 2004. Imposing essential boundary conditions in mesh-free methods. Computer Methods in Applied Mechanics and Engineering, Vol.193, pp.1257-1275. https://doi.org/10.1016/j.cma.2003.12.019

Hetenyi M., 1958. Beams on elastic foundation, The University of Michigan Press, Michigan, USA.

Liu G.R., Dai K.Y. and Thoi N.T., 2007. A smoothed finite element method for mechanics problems. Computational Mechanics, Vol.39, No.6, pp.859-877. https://doi.org/10.1007/s00466-006-0075-4

Liu G.R. and Gu Y.T., 1999. A point interpolation method. 4th Proceedings of the Asia-Pacific Conference on Computational Mechanics, Singapore, pp.1009-1014.

Liu G.R. and Chen X.L., 2001. A mesh-free method for static and free vibration analyses of thin plates of complicated shape. Journal of Sound and Vibration, Vol.241, No.5, pp.839-855. https://doi.org/10.1006/jsvi.2000.3330 
Nguyen V.H., Ton T.H.L., Chau D.T. and Dao D.N., 2017. Nonlinear static bending analysis of functionally graded plates using MISQ24 elements with drilling rotations. International Conference on Advances in Computational Mechanics, Springer, Singapore, pp.461-475. https://doi.org/10.1007/978-981-10-7149-2_31

Nyroles B., Touzot G. and Villion P., 1992. Generalizing the finite element method: diffuse approximation and diffuse elements. Computational Mechanics, Vol.10, pp.307-318. https://doi.org/10.1007/BF00364252

Puso M.A., Chen J.S., Zywicz E. and Elmer W., 2008. Meshfree and finite element nodal integration methods. International Journal for Numerical Methods in Engineering, Vol.74, pp.416-446. https://doi.org/10.1002/nme.2181

Sunitha N.V., Dodagoudar G.R. and Rao B.N., 2008. Element free Galerkin method for beams on elastic foundation. Journal of Structural Engineering, Vol.34, No.5, pp.181-188.

Ton T.H.L., Nguyen V.H. and Chau D.T., 2020. Nonlinear bending analysis of functionally graded plates using SQ4T elements based on twice interpolation strategy. Journal of Applied and Computational Mechanics, Vol. 6, No.1, pp.125-136. DOI:10.22055/jacm.2019.29270.1577

Ton T.H.L., 2020. Finite element analysis of functionally graded skew plates in thermal environment based on the new third-order shear deformation theory. Journal of Applied and Computational Mechanics, Vol.6, No.4, pp.1044-1057. DOI:10.22055/jacm.2019.31508.1881

Ton T.H.L., 2020. Improvement on eight-node quadrilateral element (IQ8) using twice-interpolation strategy for linear elastic fracture mechanics. Engineering Solid Mechanics, Vol.8, No.4, pp.323-336. DOI: 10.5267/j.esm.2020.3.005

Ton T.H.L., 2020. A novel quadrilateral element for dynamic response of plate structures subjected to blast loading. Journal of Applied and Computational Mechanics, Vol.6, No.SI, pp.1314-1323. DOI:10.22055/jacm.2020.33959.2314

Ton T.H.L., 2020. The plasticity zone at mode I crack tip. Mahasarakham International Journal of Engineering Technology, Vol.6, No.1, pp.12-16.

Trobec R., Šterk M. and Robic B., 2009. Computational complexity and parallelization of the meshless local Petrov - Galerkin method. Computers and Structures, Vol.87, pp.81-90. https://doi.org/10.1016/j.compstruc.2008.08.003

Turuna D.A., Woldaregay M.M. and Duressa G.F., 2020. Uniformly convergent numerical method for singularly perturbed convection-diffusion problems. Kyungpook Mathematical Journal, Vol.60, pp.631-647. https://doi.org/10.1002/fld.4854

Woldaregay M.M. and Duressa G.F., 2019. Parameter uniform numerical method for singularly perturbed parabolic differential difference equations. Journal of the Nigerian Mathematical Society, Vol.38, No.2, pp.223-245.

Woldaregay M.M. and Duressa G.F., 2022. Uniformly convergent numerical method for singularly perturbed delay parabolic differential equations arising in computational neuroscience. Kragujevac Journal of Mathematics, Vol.46, No.1, pp.65-84.

Wu C.T., Park C.K. and Chen J.S., 2011. A generalized approximation for the meshfree analysis of solids. International Journal for Numerical Methods in Engineering, Vol.85, pp.693-722. https://doi.org/10.1002/nme.2991

Xin L. and Chen C.S., 2004. A mesh free method using hyperinterpolation and fast fourier transform for solving differential equations. Engineering Analysis with Boundary Elements, Vol.28, pp.1253-1260. https://doi.org/10.1016/j.enganabound.2003.05.001

Zhang W., Xia M. and Liu L., 2008. Meshfree radial point interpolation method and its application for two-dimensional elastic problem. 3rd International Conference on Innovative Computing Information and Control, pp.406-408. DOI: 10.1109/ICICIC.2008.354

\section{Biographical notes}

H.T.T. Lan is currently an assistant professor at University of Architecture HCMC, Vietnam. He has more than 15 years of experience in teaching and research. His current area of research includes Meshfree Methods, Computational Mechanics, Smart Materials, and so on. He has published several papers in referred international journals. 